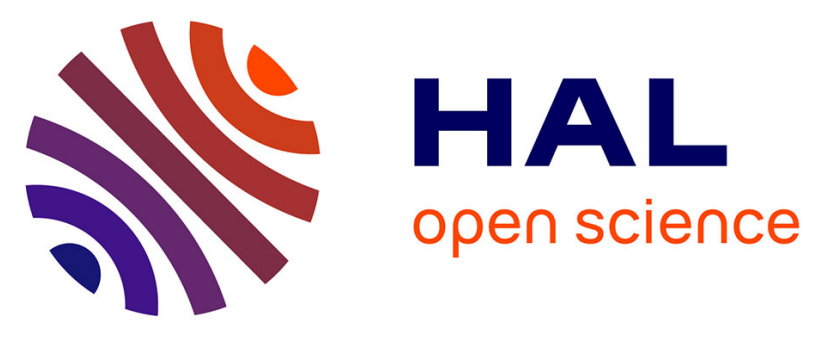

\title{
The Structural Duality of Nucleobases in Guanine Quadruplexes Controls Their Low-Energy Photoionization
}

Evangelos Balanikas, Lara Martinez-Fernandez, Roberto Improta, Peter Podbevšek, Gérard Baldacchino, Dimitra Markovitsi

\section{To cite this version:}

Evangelos Balanikas, Lara Martinez-Fernandez, Roberto Improta, Peter Podbevšek, Gérard Baldacchino, et al.. The Structural Duality of Nucleobases in Guanine Quadruplexes Controls Their Low-Energy Photoionization. Journal of Physical Chemistry Letters, 2021, 12 (34), pp.8309-8313. 10.1021/acs.jpclett.1c01846 . cea-03346313

\section{HAL Id: cea-03346313 https: / hal-cea.archives-ouvertes.fr/cea-03346313}

Submitted on 16 Sep 2021

HAL is a multi-disciplinary open access archive for the deposit and dissemination of scientific research documents, whether they are published or not. The documents may come from teaching and research institutions in France or abroad, or from public or private research centers.
L'archive ouverte pluridisciplinaire HAL, est destinée au dépôt et à la diffusion de documents scientifiques de niveau recherche, publiés ou non, émanant des établissements d'enseignement et de recherche français ou étrangers, des laboratoires publics ou privés. 


\section{Structural Duality of Nucleobases in Guanine}

\section{Quadruplexes Controls Their Low-energy}

\section{Photoionization}

Evangelos Balanikas, ${ }^{a}$ Lara Martinez-Fernandez, ${ }^{b,{ }^{*}}$ Roberto Improta, ${ }^{c}$ Peter Podbevšek, ${ }^{d,{ }^{*}}$

Gérard Baldacchino, ${ }^{a}$ and Dimitra Markovitsi ${ }^{a,}$

${ }^{a}$ Université Paris-Saclay, CEA, CNRS, LIDYL, F-91191 Gif-sur-Yvette, France

${ }^{\mathrm{b}}$ Departamento de Química, Facultad de Ciencias and Institute for Advanced Research in Chemistry (IADCHEM) Universidad Autónoma de Madrid, Campus de Excelencia UAM-CSIC, Cantoblanco, 28049 Madrid, Spain

${ }^{\mathrm{c}}$ Istituto Biostrutture e Bioimmagini- Consiglio Nazionale delle Ricerche, Via Mezzocannone 16, I-80134 Napoli, Italy

${ }^{d}$ Slovenian NMR Center, National Institute of Chemistry, Hajdrihova 19, SI-1000 Ljubljana, SloveniaIf more than one address, use symbols to match author names to address(es).

AUTHOR INFORMATION

\section{Corresponding Authors}

lara.martinez@uam.es, peter.podbevsek@ki.si,dimitra.markovitsi@cea.fr 
ABSTRACT. Guanine quadruplexes are four-stranded DNA/RNA structures composed of a guanine core (vertically stacked guanine tetrads) and peripheral groups (dangling ends and/or loops). Such a dual structural arrangement of the nucleobases favors their photoionization at energies significantly lower than the guanine ionization potential. This effect is important both in respect to the oxidative DNA damage and for applications in the field of optoelectronics. Photoionization quantum yields, determined at $266 \mathrm{~nm}$ by nanosecond transient absorption spectroscopy, strongly depend on both the type and position of the peripheral nucleobases; the highest value $\left(1.5 \times 10^{-2}\right)$ is found for the tetramolecular structure $\left(\mathrm{AG}_{4} \mathrm{~A}\right)_{4}$ in which, adenines are intermittently stacked on the adjacent guanine tetrads, as attested by NMR spectroscopy. Quantum chemistry calculations show that peripheral nucleobases interfere in a key step preceding electron ejection: charge separation, initiated by the population of charge transfer states during the relaxation of electronic excited states.

\section{TOC GRAPHICS}

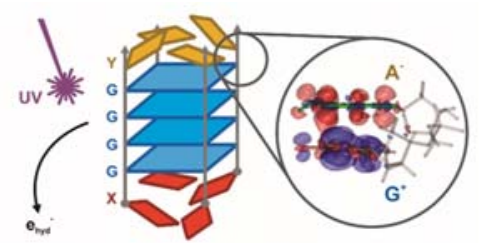

KEYWORDS. Guanine quadruplexes, DNA, photoionization, photoconductivity, oxidative damage, transient absorption spectroscopy, NMR spectroscopy, quantum chemistry. 
Guanine quadruplexes $(\mathbf{G 4})$ are four-stranded DNA/RNA structures extensively studied because of their involvement in multiple biological functions ${ }^{1-2}$ and in view of applications in the field of nanotechnology. ${ }^{3}$ In both respects, their photoionization is important. Generation of electron holes triggers chemical reactions ultimately leading to oxidative damage. ${ }^{4-5}$ Moreover, electron holes intervene in charge transport. ${ }^{6-7}$ It was reported recently ${ }^{8-9}$ that $\mathbf{G 4}$ undergo photoionization at energies significantly lower compared to the guanine vertical ionization potential. ${ }^{10}$ In contrast to high-energy photoionization, ${ }^{11-12}$ this low-energy process, also observed for other DNA systems with significantly lower efficiencies, strongly depends on the secondary structure; it could not be detected neither for mononucleotides nor for poorly stacked single strands $\left(<3 \times 10^{-4}\right) .{ }^{9,}{ }^{13}$ Here, combining spectroscopic and computational techniques, we show that the arrangement of G4 nucleobases in two distinct structural parts, vertically stacked guanine (G) tetrads, on one hand, and dangling ends and/or loops joining the tetrads, on the other, is essential in this process. Depending on their nature and position, peripheral nucleobases may be electronically coupled to guanines of the core, leading to the population of excited charge transfer $(\mathbf{C T})$ states. Thus, as positive and negative charges are located on different structural elements, charge separation, which is a key step in low-energy photoionization, is enhanced. The findings of this study should be impactful for the design of photoactive devices.

Experimentally, we studied tetramolecular G4 formed by association of four DNA strands in phosphate buffer containing $\mathrm{K}^{+}$cations. The latter, whose concentration within cells exceeds by far that of $\mathrm{Na}^{+}$ions, were shown to enhance $\mathbf{G} 4$ photoionization. ${ }^{4}{ }^{14}$ Symmetric sequences, with thymines (T), adenines (A) or AT groups at both ends, as well as asymmetric ones, with the peripheral nucleobases only at the 3 ' or the 5' ends, were examined (Table 1 and Figure 1). Their 
circular dichroism spectra, presented in the Supporting Information (SI; Figure S1), are all typical of parallel G4 as attested by a positive peak at around $264 \mathrm{~nm}$ and a negative one at $243 \mathrm{~nm} .{ }^{15}$

Table 1. One-photon ionization quantum yields ( $\phi \mathrm{i}$ ) determined for tetramolecular G4 at $266 \mathrm{~nm}$.

\begin{tabular}{cc} 
Sequence/G4 & $\phi_{\mathrm{i} \times 10^{3}}$ \\
\hline 5'-AG4A-3' & $14.8 \pm 0.5$ \\
5'-AG5A-3' & $14.4 \pm 0.1$ \\
5'-G4A-3' & $12.6 \pm 0.1$ \\
5'-AG4-3' & $9.9 \pm 0.4$ \\
5'-G4T-3' & $8.8 \pm 0.2$ \\
5'-TG4T-3' & $8.1 \pm 0.5^{14}$ \\
5'-TAG4AT-3' & $7.0 \pm 0.51$ \\
5'-TG4-3' & $4.7 \pm 0.7$
\end{tabular}

The propensity of the examined G4 to undergo low-energy photoionization was evaluated by their one-photon ionization quantum yields $\phi_{\mathrm{i}}$, determined using nanosecond transient absorption spectroscopy with excitation at $266 \mathrm{~nm}$. Briefly, the decays of the hydrated ejected electrons (Figure S2) were recorded for various excitation intensities. ${ }^{9}$ Subsequently, ionization curves were constructed and $\phi_{\mathrm{i}}$ values derived from the intercept on the vertical axes (Figures 2 and S3).

The $\phi_{\mathrm{i}}$ values found for $\mathbf{G} \mathbf{4}$ composed of four G-tetrads exhibit important variations, up to a factor 3 (Table 1). Replacing A by $\mathrm{T}$ induces a decrease in $\phi \mathrm{i}$, whereas their positioning at the 3 ' ends leads to significantly higher values compared to 5 ' ends analogs. In contrast, adding an extra Gtetrad has no effect, the $\phi_{\mathrm{i}}$ values of $5^{\prime}-\mathrm{AG}_{4} \mathrm{~A}-3^{\prime}$, and $5^{\prime}-\mathrm{AG}_{5} \mathrm{~A}-3^{\prime}$ ' being practically the same. Consequently, it appears that the synergy between central core and peripheral groups plays an important role in the propensity of $\mathbf{G 4}$ to undergo low-energy photoionization. 

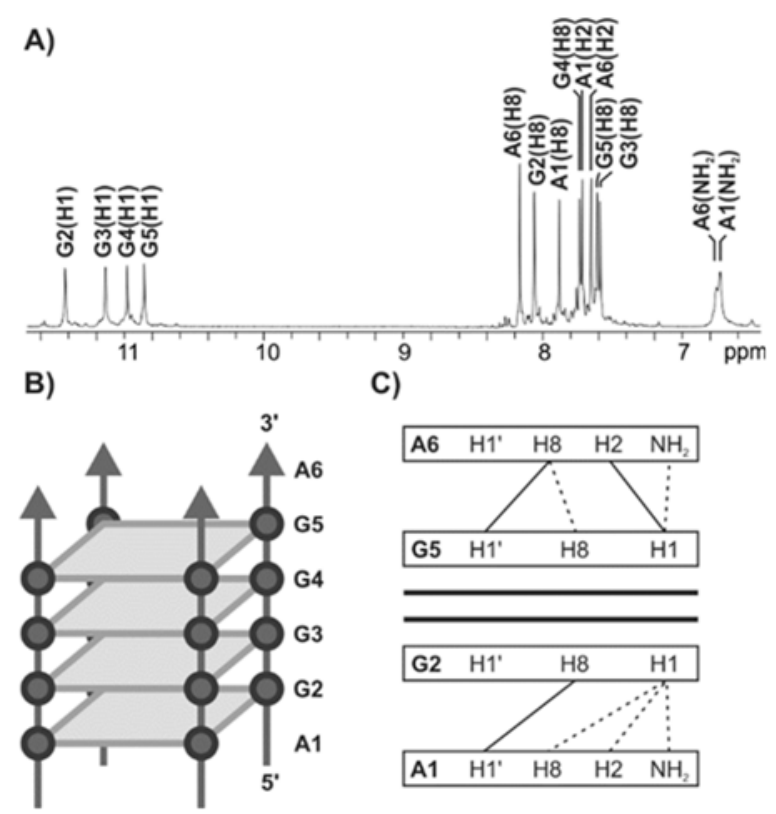

C)

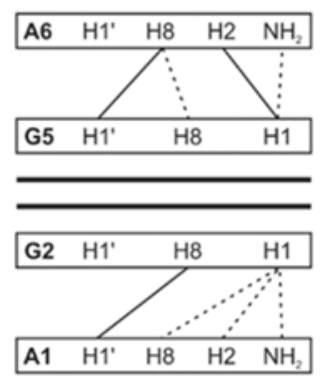

Figure 1. Structural properties of 5'-AG4A-3'. A) Imino, aromatic and amino region of the 1D 1H NMR spectrum. B) Schematic structure of parallel tetramolecular G4. C) Representation of NOE cross-peaks between outer G-tetrads and flanking adenines extracted from a 2D NOESY spectrum $\left(\tau_{\mathrm{m}}=200 \mathrm{~ms}\right)$. Dashed lines represent weak cross-peaks.

5'-AG4A-3' exhibits the highest $\phi_{\mathrm{i}}\left(1.5 \times 10^{-2}\right)$ determined so far for any DNA system. It is an order of magnitude larger than that of genomic DNA, whose ionization curve is shown in Figure 2 for comparison. Moreover, it is twice as high compared to 5'-TG4T-3'. The difference between the $\phi_{\mathrm{i}}$ of these two G4 scaffolds could possibly arise from formation of A-tetrads. As a matter of fact, NMR and crystallographic studies reported that in $5^{\prime}-\mathrm{TG}_{4} \mathrm{~T}-3$ ' the position of thymines is either poorly defined or they are rotated away from the $\mathbf{G} \mathbf{4}$ core. ${ }^{16-17}$ But adenines in some $\mathbf{G} \mathbf{4}$ were found to self-associate via Hoogsteen bonding. ${ }^{18-20}$ 


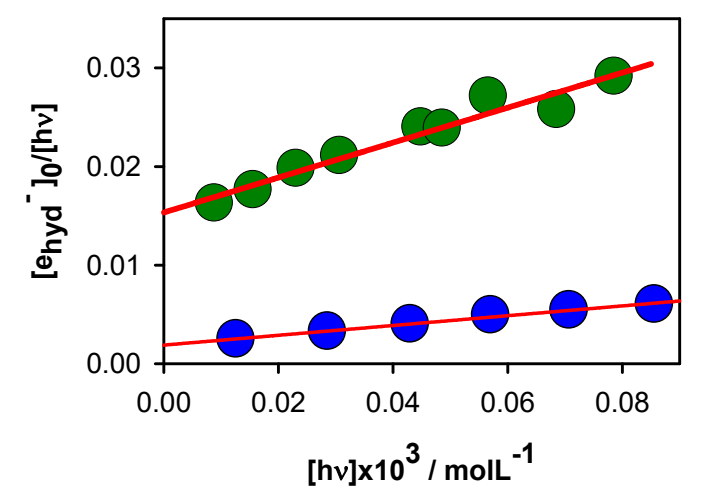

Figure 2. Ionization curves determined for 5'-AG4A-3' (green) and duplex calf thymus DNA (blue) with $266 \mathrm{~nm}$ excitation; [ $\left.\mathrm{e}_{\mathrm{hyd}}{ }^{-}\right]_{0}$ and $[\mathrm{hv}$ ] denote, respectively, the concentration of hydrated ejected electrons and absorbed photons per laser pulse. Experimental points (circles) are fitted with linear functions $\left[\mathrm{ehyd}^{-}\right] 0 /[\mathrm{h} v]=\phi_{\mathrm{i}}+\alpha[\mathrm{h} v]$ (red).

In order to investigate the above question, we utilized solution NMR. Although 1D NMR of 5'AG4A-3' have been reported, ${ }^{21}$ no detailed structural study was carried out. The $1 \mathrm{D}{ }^{1} \mathrm{H}$ NMR spectrum of $5^{\prime}-\mathrm{AG}_{4} \mathrm{~A}-3^{\prime}$ ' revealed four well-resolved resonances in the $\delta$ range 10.8-11.5 ppm (Figure 1A); they are indicative of $\mathrm{G}$ imino protons involved in Hoogsteen hydrogen bonding, suggesting the formation of symmetric tetrameric G4 (Figure 1B). Several sets of low-intensity signals $(<5 \%)$ were also observed; they possibly correspond to alternative folds. Resonances of the major species could be assigned through sequential connectivities in 2D NOESY NMR spectra (Figure S4). Based on NOE connectivities and intensities of intranucleotide $\mathrm{H} 1{ }^{\prime}{ }_{n}-\mathrm{H} 8_{\mathrm{n}}$ cross-peaks all nucleotides were assigned the anti glycosidic conformation. Interestingly, A1 and A6 nucleotides exhibit observable broad resonances at $\delta 6.75$ and $6.77 \mathrm{ppm}$, respectively, indicating that at $25{ }^{\circ} \mathrm{C}$ amino protons are partially protected from exchange with solvent. However, amino proton pairs of A1 and A6 are isochronous, thus precluding formation of long-lived hydrogen bonds. No down-field amino resonances, which would suggest hydrogen bonding of adenines with 
adjacent adenines or guanines, could be observed at temperatures as low as $0{ }^{\circ} \mathrm{C}$. Nevertheless, several NOE cross-peaks were observed between G of the outer G-tetrads and flanking A (Figure 1C). Most notably, amino protons of A1 and A6 exhibit NOE cross-peaks with imino protons of G2 and G5, respectively, which suggest that in a time-averaged manner A1 and A6 nucleobases are spatially close to $\mathrm{G}$ of adjacent G-tetrads.

In conclusion, NMR measurements, have not detected hydrogen bonding between adenines, showing that no stable A-tetrads are formed. Nevertheless, intermittent stacking of individual adenines is likely. Furthermore, adenines could form heterogenous capping structures, protecting their amino proton from exchange with solvent.

Having ruled out the specific role of A-tetrads, we examined whether CT states may be formed between a peripheral nucleobase and a $\mathrm{G}$ of the neighboring tetrad. To this end, we first considered a G-tetrad with an adenine at the 3' position (5'-G-tetradA-3'; Figure S6A). The relative position between $\mathrm{G}$ and $\mathrm{A}$ is comparable to previously reported structures (Figure S6B). ${ }^{22}$ Results obtained for this system are quite similar with those computed for the 5'-GA-3' stacked dinucleotide, except for the presence of additional $\pi \pi^{*} \mathrm{G}$ states, resulting from the larger the number of $\mathrm{G}$ (Tables $\mathrm{S} 1$ to S4). Consequently, we compared the sequence effect on the electronic excited states of stacked dinucleotides: 5'-AG-3', 5'-GA-3', 5'-TG-3' and 5'-GT-3'.

We considered at quantum mechanical (QM) level the entire system (two nucleobases, the connecting backbone and the counter ion), embedded in water through a polarizable continuum model. We used the density functional theory, in particular the M052X functional, and its timedependent version (TD-DFT) combined with the 6-31G(d) basis set. This method was selected due to its good performance in comparable studies. ${ }^{23}$ We characterized the Franck-Condon (FC) excited states and, then, studied their relaxation pathways. 
Important differences among the four dinucleotides appear already at the FC region (Table S1 and Figure S7). We found that in 5'-GA-3' the coupling between the bright and CT states is larger. Indeed, its spectroscopic state $\mathrm{S}_{2}$ has some $\mathbf{C T}$ character $(0.3$ a.u.). In contrast, this state is completely devoid of $\mathbf{C T}$ character in 5'-AG-3'. Instead, 5'-AG-3' presents a low-energy state with an oscillator strength of $2 \times 10^{-1}$ and significant $\mathrm{G}^{+} \rightarrow \mathrm{A}^{-} \mathbf{C T}$ character $(0.6$ a.u.) but whose energy difference with respect to the spectroscopic state is $\sim 0.3 \mathrm{eV}$. In addition, the energy of higher CT states $\left(\mathrm{S}_{9}\right)$ is $\sim 0.1 \mathrm{eV}$ lower in 5'-GA-3' compared to 5'-AG-3'. For the T analogs, 5'GT-3' has a clear low-lying CT state (0.8 a.u.), but with very small oscillator strength $\left(10^{-2}\right)$, whereas in 5'-TG-3' the first state with significant CT character is higher in energy $(+0.5 \mathrm{eV})$. Our finding that CT states involving transfer of an electron towards the $3^{\prime}$ ' end are more stable than in the opposite direction, agrees with previous reports for other types of dinucleotides. ${ }^{24-27}$
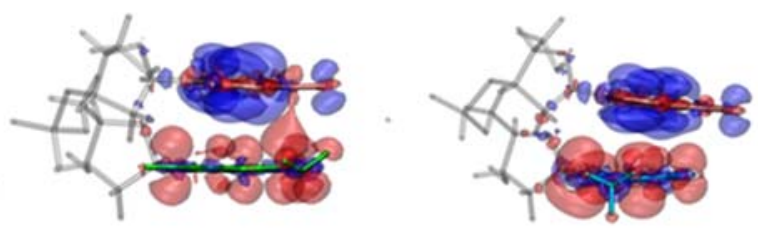

Figure 3. Excited state CT minima and the associated electronic density difference computed for 5'-GA-3' (left) and 5'-GT-3' (right). Color code: guanine (pink), adenine (green), thymine (cyan), backbone (silver), hole (blue) and electron (red).

Coming to the excited state relaxation, we examined the population of $\mathbf{C T}$ states. To this end, we optimized the lowest five excited states as well as the first state with clear $\mathrm{G}^{+} \rightarrow \mathrm{T}^{-} / \mathrm{A}^{-} \mathbf{C T}$ character, locating different types of minima. Their stabilities and geometrical features are 
presented in Table S3 and Figure S8. 5'-GA-3' presents a minimum with important CT character that can be reached from the bright $S_{2}$ or the $S_{1}$ state and whose stability is comparable to that of the $\pi \pi^{*}$ (La G)-minimum (Figure 3, Table S3). 5'-AG-3' and 5'-GT-3' exhibit also such stable CT minima resulting from higher and/or dark excited states ( $\mathrm{S}_{9}$ and $\left.\mathrm{S}_{4}\right)$. In contrast, in the case of 5'-TG-3', none of the optimizations lead to a CT minimum.

Our computational results show that the probability of populating a CT state during the excited state relaxation is higher if the "peripheral" nucleobase is located in the 3', which matches the ordering of $\phi_{\mathrm{i}}$ (Table 1). But CT population in $\mathbf{G 4}$ does not depend only on electronic factors but also on the stacking probability. However, as NMR spectra did not reveal asymmetric stacking of the peripheral nucleobases, electronic factors should prevail. In this respect, we remark that the steady-state absorption spectra of 5'-G4T-3' and 5' $-\mathrm{TG}_{4}-3$ ' are practically identical (Figure S5). Yet their $\phi_{\mathrm{i}}$ differ by a factor 2 , pinpointing the role of the excited state relaxation. Within this scheme, the lower $\phi_{i}$ value of 5'-TAG4AT-3' compared to $5^{\prime}-\mathrm{AG}_{4} \mathrm{~A}-3^{\prime}$ ' is explained by both a smaller stacking probability of adenines when attached to thymines and interaction between them during the relaxation process. ${ }^{26}$ Other factors, such as competition between $\mathrm{A}^{+} \rightarrow \mathrm{T}^{-}$and $\mathrm{G}^{+} \rightarrow \mathrm{A}^{-}$ CT states cannot be excluded. ${ }^{28}$

Our QM calculations provided a qualitative interpretation for the role of peripheral groups. A quantitative description, encompassing electronic relaxation and conformational motions in the entire G4, could be achieved by integrating molecular dynamics simulations. Such an approach, already applied for the determination of FC states in small $\mathbf{G 4},{ }^{29}$ should become affordable in the near future for the study of dynamical processes in larger systems.

Femtosecond studies detected the population of $\mathbf{C T}$ states in telomeric $\mathbf{G 4} \cdot{ }^{30-32}$ It was also shown that the excited state relaxation pattern remains the same in reversed micelles, mimicking the 
crowding conditions in cells. ${ }^{32}$ QM calculations performed for such G4 showed that CT states may be formed between a $\mathrm{T}$ in the loop and a $\mathrm{G}$ in the core, but experiments have not disentangled this type of mixed CT states from those involving two guanines. ${ }^{31}$ More importantly, the study on intrinsic G4 fluorescence clearly evidenced the existence of interactions between the G core and nucleobases in loops and dangling groups. ${ }^{33-34}$

Our comparative study on tetramolecular $\mathbf{G 4}$ formed in the presence of $\mathrm{K}^{+}$cations and characterized by a parallel arrangement of $\mathrm{G}$, unveiled the importance of the peripheral nucleobases for their low-energy photoionization. Comparing the $\phi_{\mathrm{i}}$ values reported previously for monomolecular telomeric G4 formed in the presence of $\mathrm{Na}^{+}$cations and characterized by antiparallel arrangement of $\mathrm{G}$, we realize retrospectively that a similar effect takes place: when the dangling groups TA at the 5' end and TT at the 3' end are removed from the telomeric sequence $\phi_{\mathrm{i}}$ decreases from $5.2 \times 10^{-3}$ to $4.5 \times 10^{-3},{ }^{9}$ despite the fact that the G percentage increases from $48 \%$ to $57 \%$.

Duplexes are devoid of the structural duality characterizing G4. Yet, they do undergo lowenergy photoionization $\left(\phi_{i} \leq 2 \times 10^{-3}\right) \cdot{ }^{9}$ In this case, inhomogeneity is introduced through conformational disorder and also base sequence. Thus, repetitive sequences (homopolymeric AT, alternating AT or alternating GC), exhibit quite similar $\phi_{\mathrm{i}}$. But $\phi_{\mathrm{i}}$ increases by $c a .40 \%$ in systems containing GGG steps. ${ }^{9}$ The latter, due to their low oxidation potential, behave as traps for electron holes. ${ }^{35-37}$ Accordingly, the trapping of the positive charge is expected to be particularly efficient in G4, where it may be delocalized. ${ }^{23,37-38}$ Eventually, electron ejection occurs from the peripheral nucleobase bearing the negative charge, whose vertical ionization potential is lower compared to the "neutral" analog. ${ }^{39}$ 
Beyond DNA, the existence of distinct structural units is a prerequisite for applications relying on photoinduced charge transfer, as artificial photosynthesis. ${ }^{40-41}$ In addition, electron photodetachment is closely related to photoconductivity and photoelectrochemistry, used, among others, for bioanalysis. Sensors involving G4 associated with external photoactive molecules have been reported. ${ }^{42-43}$ The discovery of the low-energy $\mathbf{G 4}$ photoionization and the characterization of structural and electronic factors governing this phenomenon presented here open new perspectives in this field through appropriate design of peripheral groups (for example, by joining G4 at their 5', as proposed for DNAzymes). ${ }^{21}$

\section{ASSOCIATED CONTENT}

Supporting Information Available. Experimental Details; Additional Experimental Results; Additional Computational Results.

\section{AUTHOR INFORMATION}

\section{Notes}

The authors declare no competing financial interests.

\section{ACKNOWLEDGMENT}

This work has received funding from the European Union's Horizon 2020 research and innovation programme under the Marie Sklodowska-Curie grant agreement No. 765266 (LightDyNAmics). L.M.F. thanks the PID2019-110091GB-I00 (MICINN) and GV/2020/226 (Generalitat Valenciana) projects for financial support and the Centro de Computación Científica UAM (CCC-UAM) for computing time.

\section{REFERENCES}


1. Rhodes, D.; Lipps, H. J. G-quadruplexes and their regulatory roles in biology. Nucleic Acids Res. 2015, 43, 8627-8637.

2. Kharel, P.; Becker, G.; Tsvetkov, V.; Ivanov, P. Properties and biological impact of RNA G-quadruplexes: from order to turmoil and back. Nucleic Acids Res. 2020, 48, 12534-12555.

3. Mergny, J. L.; Sen, D. DNA Quadruple Helices in Nanotechnology. Chem. Rev. 2019, $119,6290-6325$.

4. Cadet, J.; Douki, T.; Ravanat, J. L. Oxidatively generated damage to the guanine moiety of DNA: Mechanistic aspects and formation in cells. Acc. Chem. Res. 2008, 41, 1075-1083.

5. Delaney, S.; Barton, J. K. Charge transport in DNA duplex/quadruplex conjugates. Biochemistry 2003, 42, 14159-14165.

6. Livshits, G. I.; Stern, A.; Rotem, D.; Borovok, N.; Eidelshtein, G.; Migliore, A.; Penzo, E.; Wind, S. J.; Di Felice, R.; Skourtis, S. S.; Carlos Cuevas, J.; Gurevich, L.; Kotlyar, A. B.; Porath, D. Long-range charge transport in single G-quadruplex DNA molecules. Nat. Nanotechnol. 2014, 9, 1040-1046.

7. Sha, R. J.; Xiang, L. M.; Liu, C. R.; Balaeff, A.; Zhang, Y. Q.; Zhang, P.; Li, Y. Q.; Beratan, D. N.; Tao, N. J.; Seeman, N. C. Charge splitters and charge transport junctions based on guanine quadruplexes. Nat. Nanotechnol. 2018, 13, 316-321.

8. Banyasz, A.; Martinez-Fernandez, L.; Balty, C.; Perron, M.; Douki, T.; Improta, R.; Markovitsi, D. Absorption of Low-Energy UV Radiation by Human Telomere G-Quadruplexes Generates Long-Lived Guanine Radical Cations. J. Am. Chem. Soc. 2017, 139, 10561-10568. 9. Balanikas, E.; Banyasz, A.; Douki, T.; Baldacchino, G.; Markovitsi, D. Guanine Radicals Induced in DNA by Low-Energy Photoionization. Acc. Chem. Res. 2020, 53, 1511-1519. 
10. Schroeder, C. A.; Pluharova, E.; Seidel, R.; Schroeder, W. P.; Faubel, M.; Slavicek, P.; Winter, B.; Jungwirth, P.; Bradforth, S. E. Oxidation Half-Reaction of Aqueous Nucleosides and Nucleotides via Photoelectron Spectroscopy Augmented by ab Initio Calculations. J. Am. Chem. Soc. 2015, 137, 201-209.

11. Candeias, L. P.; O'Neill, P.; Jones, G. D. D.; Steenken, S. Ionization of polynucleotides and DNA in aqueous solution by $193 \mathrm{~nm}$ pulsed laser light: identification of base derived radicals. Int. J. Radiat. Biol. 1992, 61, 15-20.

12. Melvin, T.; Plumb, M. A.; Botchway, S. W.; Oneill, P.; Parker, A. W. 193 nm Light Induces Single-strand Breakage of DNA Predominantly at Guanine. Photochem. Photobiol. 1995, 61, 584-591.

13. Balanikas, E.; Markovitsi, D. DNA photoionization: from high to low energies. In DNA Photodamage, Importa, R.; Douk, T., Eds. RSC: Cambridge, 2021; p in press.

14. Behmand, B.; Balanikas, E.; Martinez-Fernandez, L.; Improta, R.; Banyasz, A.; Baldacchino, G.; Markovitsi, D. Potassium Ions Enhance Guanine Radical Generation upon Absorption of Low-Energy Photons by G-quadruplexes and Modify Their Reactivity. J. Phys. Chem. Lett. 2020, 11, 1305-1309.

15. Karsisiotis, A. I.; Hessari, N. M.; Novellino, E.; Spada, G. P.; Randazzo, A.; da Silva, M. W. Topological Characterization of Nucleic Acid G-Quadruplexes by UV Absorption and Circular Dichroism. Angew. Chem. Int. Ed. 2011, 50, 10645-10648.

16. Aboul-ela, F.; Murchie, A. I. H.; Norman, D. G.; Lilley, D. M. J. Solution structure of a parallel-stranded tetraplex formed by d(TG4T) in the presence of sodium ions by nuclear magnetic resonance spectroscopy. J. Mol. Biol. 1994, 243, 458-471. 
17. Phillips, K.; Dauter, Z.; Murchie, A. I. H.; Lilley, D. M. J.; Luisi, B. The crystal structure of a parallel-stranded guanine tetraplex at 0.95 angstrom resolution. J. Mol. Biol. 1997, 273, 171182.

18. Patel, P. K.; Ainavarapu, R. K.; Hosur, R. V. NMR studies on truncated sequences of human telomeric DNA: observation of a novel A-tetrad. Nucleic Acids Res. 1999, 27, 3836-3843. 19. Searle, M. S.; Williams, H. E. L.; Gallagher, C. T.; Grant, R. J.; Stevens, M. F. G. Structure and $\mathrm{K}+$ ion-dependent stability of a parallel-stranded DNA quadruplex containing a core A-tetrad. Org. Biomol. Chem. 2004, 2, 810-812.

20. Pan, B. C.; Xiong, Y.; Shi, K.; Deng, J. P.; Sundaralingam, M. Crystal structure of an RNA purine-rich tetraplex containing adenine tetrads: Implications for specific binding in RNA tetraplexes. Structure 2003, 11, 815-823.

21. Virgilio, A.; Esposito, V.; Lejault, P.; Monchaud, D.; Galeone, A. Improved performances of catalytic G-quadruplexes (G4-DNAzymes) vi a the chemical modifications of the DNA backbone to provide G-quadruplexes with double 3 '-external G-quartets. Int. J. Biol. Macromol. 2020, 151, 976-983.

22. Yang, D. G-Quadruplex DNA and RNA. In G-Quadruplex Nucleic Acids: Methods and Protocols, Yang, D.; Lin, C., Eds. Humana: New York, 2019; pp 1-24.

23. Martinez-Fernandez, L.; Esposito, L.; Improta, R. Studying the excited electronic states of guanine rich DNA quadruplexes by quantum mechanical methods: main achievements and perspectives. Photochem. Photobiol. Sci. 2020, 19, 436-444.

24. Aquino, A. J. A.; Nachtigallova, D.; Hobza, P.; Truhlar, D. G.; Hattig, C.; Lischka, H. The Charge-Transfer States in a Stacked Nucleobase Dimer Complex: A Benchmark Study. J. Comput. Chem. 2011, 32, 1217-1227. 
25. Santoro, F.; Barone, V.; Lami, A.; Improta, R. The excited electronic states of adenineguanine stacked dimers in aqueous solution: a PCM/TD-DFT study. Phys. Chem. Chem. Phys. 2010, 12, 4934-4948.

26. Martinez-Fernandez, L.; Improta, R. Novel adenine/thymine photodimerization channels mapped by PCM/TD-DFT calculations on dApT and TpdA dinucleotides. Photochem. Photobiol. Sci 2017, 16, 1277-1283.

27. Martinez-Fernandez, L.; Improta, R. Sequence dependence on DNA photochemistry: a computational study of photodimerization pathways in $\mathrm{TpdC}$ and $\mathrm{dCpT}$ dinucleotides. Photochem. Photobiol. Sci 2018, 17, 586-591.

28. Martinez-Fernandez, L.; Zhang, Y. Y.; de la Harpe, K.; Beckstead, A. A.; Kohler, B.; Improta, R. Photoinduced long-lived charge transfer excited states in AT-DNA strands. Phys. Chem. Chem. Phys. 2016, 18, 21241-21245.

29. Avagliano, D.; Tkaczyk, S.; Sanchez-Murcia, P. A.; Gonzalez, L. Enhanced Rigidity Changes Ultraviolet Absorption: Effect of a Merocyanine Binder on G-Quadruplex Photophysics. J. Phys. Chem. Lett. 2020, 11, 10212-10218.

30. McGovern, D. A.; Quinn, S.; Doorley, G. W.; Whelan, A. M.; Ronayne, K. L.; Towrie, M.; Parker, A. W.; Kelly, J. M. Picosecond infrared probing of the vibrational spectra of transients formed upon UV excitation of stacked G-tetrad structures. Chem. Comm. 2007, 51585160.

31. Martinez-Fernandez, L.; Changenet, P.; Banyasz, A.; Gustavsson, T.; Markovitsi, D.; Improta, R. A Comprehensive Study of Guanine Excited State Relaxation and Photoreactivity in G-Quadruplexes. J. Phys. Chem. Lett. 2019, 10, 6873-6877. 
32. Ma, C. S.; Chan, R. C.-T.; Chan, C. T.-L.; Wong, A. K.-W.; Kwok, W.-M. Real-time Monitoring Excitation Dynamics of Human Telomeric Guanine Quadruplexes: Effect of Folding Topology, Metal Cation, and Confinement by Nanocavity Water Pool. J. Phys. Chem. Lett. 2019, $10,7577-7585$.

33. Kwok, C. K.; Sherlock, M. E.; Bevilacqua, P. C. Effect of Loop Sequence and Loop Length on the Intrinsic Fluorescence of G-Quadruplexes. Biochemistry 2013, 52, 3019-3021. 34. Gustavsson, T.; Markovitsi, D. Fundamentals of the Intrinsic DNA Fluorescence. Acc. Chem. Res. 2021, 54, 1226-1235.

35. Meggers, E.; Michel-Beyerle, M. E.; Giese, B. Sequence dependent long range hole transport in DNA. J. Am. Chem. Soc. 1998, 120, 12950-12955.

36. Saito, I.; Nakamura, T.; Nakatani, K.; Yoshioka, Y.; Yamaguchi, K.; Sugiyama, H. Mapping of the hot spots for DNA damage by one-electron oxidation: Efficacy of GG doublets and GGG triplets as a trap in long-range hole migration. J. Am. Chem. Soc. 1998, 120, 1268612687.

37. Kumar, A.; Adhikary, A.; Sevilla, M. D.; Close, D. M. One-electron oxidation of ds(5 'GGG-3 ') and ds(5 '-G(8OG)G-3 ') and the nature of hole distribution: a density functional theory (DFT) study. Phys. Chem. Chem. Phys. 2020, 22, 5078-5089.

38. Sun, W. M.; Varsano, D.; Di Felice, R. Effects of G-Quadruplex Topology on Electronic Transfer Integrals. Nanomaterials 2016, 6 .

39. Schiedt, J.; Weinkauf, R.; Neumark, D. M.; Schlag, E. W. Anion spectroscopy of uracil, thymine and the amino-oxo and amino-hydroxy tautomers of cytosine and their water clusters. Chem. Phys. 1998, 239, 511-524. 
40. Romero, E.; Novoderezhkin, V. I.; van Grondelle, R. Quantum design of photosynthesis for bio-inspired solar-energy conversion. Nature 2017, 543, 355-365.

41. Kranz, C.; Wachtler, M. Characterizing photocatalysts for water splitting: from atoms to bulk and from slow to ultrafast processes. Chem. Soc. Rev. 2021, 50, 1407-1437.

42. Liu, T. L.; Sun, D. X.; Gu, M. M.; Wu, X. M.; Wang, G. L. Intercalative methylene blue as an efficient signal molecule of tremella-like Bi 2 WO 6: Toward high performance photoelectrochemical biosensing. Sens. Actuators B Chem. 2020, 317.

43. Gu, M.; Sun, D.; Liu, T.; Wu, X.; Wang, G.-L. Homogenous cathodic photoelectrochemistry for DNA binding protein analysis. Sens. Actuators B Chem. 2020, 324 